\title{
The Exact Solution of Spatial Logit Response Games ${ }^{1}$
}

\author{
Tomohiko Konno ${ }^{2}$ and Yannis M. Ioannides ${ }^{3}$
}

\begin{abstract}
This paper proposes a logit response game with a spatial social structure and solves it exactly. We derive closed-form solutions for the strategy choice probabilities, the spatial correlation function of strategies of distant players, and the expected utility. We study how the probability of adopting a cooperative strategy in a prisoner's dilemma game and the probability of adopting Pareto efficient strategies in a cooperation game are affected by changes in the parameter that expresses payoff-responsiveness.
\end{abstract}

Keywords: Spatial Logit Game, Exact solution, Network,Logit Local Interaction, Competition with Spatial Structure, Spatial coordination game

JEL: R00, R30, R32, R39, D21

\section{Introduction}

Almost all the economic and social activities involve spatial proximity. A retailer, a supermarket, a convenience store, a restaurant, etc, compete with their neighbors. A retailer engages in price competition with its neighbors. A restaurant decides whether or not to renovate taking account of the competition with its neighboring restaurants. One chooses whether to open a shop at a location or not, taking consideration of the competition with its neighbors if they exist. Spatial proximity is not limited to economic activities only. For example, patterns of diffusion and changes of European languages and cultures can be also analyzed as spatial coordination games. Changes and mutual influences propagate spatially.

We analyze the decision problems involving interactions over space by investigating a spatial game in which players play games only with their neighbors and choose discrete strategies according to the logit response model (Luce (1959); Anderson et al. (1992); Train (2003)). The associated logit probabilities are endogenous responses that express that players are not perfectly rational. Their evaluation of the payoffs of their actions is subject to error.

The specific model that we will study in the present paper is a simple spatial logit response two-strategy game with general symmetric payoffs. Several previous authors have studied local interactions models of the logit type that we address in this paper. Several authors have drawn attention to the fact that it is mathematically equivalent to the Ising model, which was

\footnotetext{
${ }^{1}$ The current version became possible after the authors became aware of each other's papers, Ioannides of Konno's original paper and Konno of Ioannides's 2004 conference version of his Economic Theory (2006). Ioannides (2004) includes results much along the lines of Konno's working paper, which had not been included in the published version of Ioannides (2006). Thereupon they decided to join forces and combine results from both papers into the current paper.

${ }^{2}$ National Institute of Information and Communications Technology tomo.konno@gmail.com

${ }^{3}$ Department of Economics, Tufts University, Medford, MA 02155, USA yannis.ioannides@tufts.edu
} 
introduced by statistical physics (Ising (1925); Baxter (1982); Brézin (2010); Nishimori and Ortiz (2011)), and has been adopted by numerous econophysics applications (Weidlich (1972, 1991); Sznajd-Weron and Sznajd (2000)). Models relying on the machinery of random fields were first introduced by Föllmer, and then adopted by others (Föllmer (1974); Blume (1993); Lux (1995, 1997); Brock and Durlauf (2001); Hautsch and Klotz (2003); Bayer and Timmins (2005); Ioannides (2006); Zanella (2007); Chang (2007); Levy (2008)). However, Blume must be credited with recognizing that Gibbs distributions admit a (spatial) Logit interpretation.

The progress to date has been incomplete. Many applications emphasize steady-state properties and rely on mean-field approximations or numerical simulations. The mean-field approximation does not account for the richness associated with the underlying spatial structure. Mean-field approximations simply fall short of the exact solution in closed-form proposed by the present paper. It is thus attractive to take full advantage of the techniques developed by statistical physics to obtain exact solutions. Although numerical simulations afford us some intuition, exact solutions helps us to understand the models in full depth.

In the present paper, we adapt the model to the requirements of Glauber dynamics of the one-dimensional Ising model (Glauber (1963)) and derive the exact solution of the steady state employing statistical physics methods. We will derive exact solutions for the probabilities that a player chooses different strategies, the strategy correlation function $C(r)$ (the effect of a strategy chosen by a player on the strategies chosen by the players at a graph-distance $r$ ), and the expected utility of the decision process of the typical player. We show how the probability of adopting a cooperative strategy in a prisoner's dilemma game and the probability of adopting a Pareto efficient strategy in a cooperation game, respectively, change in response to changes in $\beta$, the payoff-responsiveness parameter that reflects the dispersion of the underlying stochastic shocks that affect player valuations. We also derive the probabilities in the limit as $\beta$ goes infinity.

Having addressed the solution to spatial logit response games, we show that there exists a formal similarity with binary discrete choice models with social interactions. Taking off from Brock and Durlauf (2001), Ioannides (2004, 2006) proposed an interactive discrete choice model, with agents' acting under full information of other agents' decisions.

\subsection{Outline of the Paper}

The remainder of the present paper goes as follows. Section 2 introduces logit response games embedded in spatial structures, and Section 3 gives the exact solution. We exactly derive the probability that a player chooses a particular strategy, the strategy correlation function, and the expected utility $E[U]$. We also discuss the relationship between the mean-field solution and the exact ones. Section 4 examines the dependence of the probability of adopting a cooperative strategy in a prisoner's dilemma game and the probability of adopting a Pareto efficient strategy in cooperation game, respectively, to changes in the payoff-responsiveness parameter, $\beta$. Section 5 discusses the conceptual and analytical similarities between the treatment herein of spatial logit response games and interactive discrete choice models. Section 6 concludes.

Appendices A, B, and $\mathrm{C}$ explain the method of deriving the exact solution, which relies on statistical physics, in further detail. Appendix A discusses the detailed balance condition

and then derives the strategy probabilities in the steady state, which is interpreted as an equilibrium. Appendix B derives the solution by the transfer-matrix method. Appendix C 
shows that our results do not depend on whether the spatial structure is a circle or a line in the limit with many agents.

\section{The Model}

Let $N$ players be located on a circle, as illustrated in Fig. 1. Player $j$ interacts with his neighbors only and derives payoff from each game outcome. As illustrated in Fig. 1, player $j$ 's neighbors are players $j-1$ and $j+1$. We assume the periodic boundary condition: player $N+1$ is the player 1 and player $k+N$ is the player $k$. We show in Appendix $\mathrm{C}$ that in the limit of many players, the solution does not depend on whether the spatial structure is a circle or a line. The players can not move to other locations. We justify this by assuming that it is too costly for a player to move, once the location has been determined, although it is costless to engage in a strategic game with his neighbors. However, in principle, one may extend the model and assume that to exit, or to enter, is indeed a strategy. The payoff matrix of the game is given by the general form

$$
\begin{gathered}
A \\
A \\
B\left(\begin{array}{cc}
(a, a) & (b, c) \\
(c, b) & (d, d)
\end{array}\right) .
\end{gathered}
$$

At each time step, one randomly chosen player chooses a strategy from the strategy space $\{A, B\}$, according to a logit probability, conditional on the strategies of their neighbors. Allowing for the players to update their strategies sequentially is no loss of generality and may be formalized by means of the device of a Poisson clock; c.f., Blume (1993).

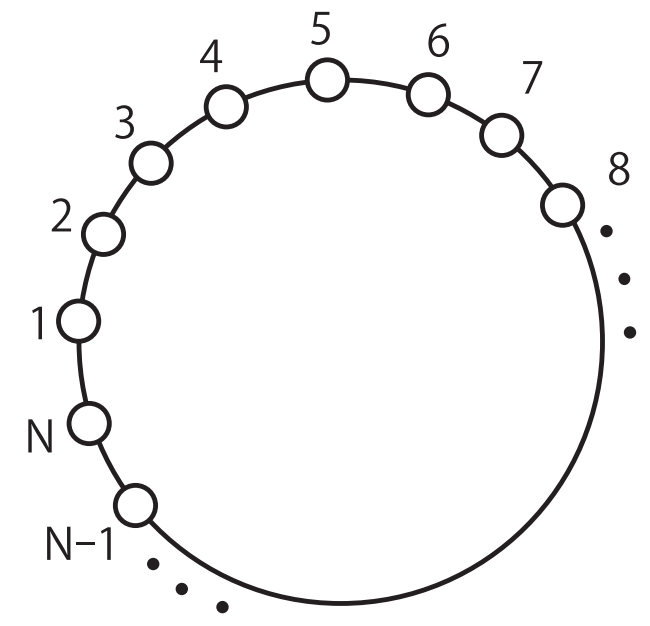

Figure 1: The players are set on a circle and play games with the adjacent players only. The numbers identify the players.

In order to take advantage of the machinery of the Ising model of statistical physics, we 
transform the matrix (1) by defining the auxiliary variables $(x, y, z, w)$ as follows:

$$
\begin{aligned}
& x=\frac{a-b-c+d}{4}, \\
& y=\frac{a+b-c-d}{4}, \\
& z=\frac{a-b+c-d}{4}, \\
& w=\frac{a+b+c+d}{4} .
\end{aligned}
$$

Let $S_{i}$ denote the strategy of player $i ; S_{i}=+1$ corresponds to the strategy $A$ and $S_{i}=-1$ corresponds to the strategy $B$. Suppose that player $i$ and player $j$ are adjacent to each other; in other words, $j=i-1$ or $i+1$. The payoff $U_{i, j}$ for player $i$ from strategy $S_{i}$ when player $j$ adopts strategy $S_{j}$ is expressed in terms of $x, y, z$, and $w$ as follows:

$$
U_{i, j}=x S_{i} S_{j}+y S_{i}+z S_{j}+w .
$$

By substituting $S_{i}= \pm 1$ and $S_{j}= \pm 1$ in Eq. (3), we can confirm that the payoff $U_{i, j}$ reproduces the payoff matrix in Eq. (1). What a player $i$ chooses when choosing strategy is the value of $S_{i}$. Let $N$-vector $\mathcal{S}$ denote the strategies of all the players $\left\{S_{1}, S_{2}, \cdots, S_{N}\right\}$. Let $\partial i$ denote the set of all players that are $i$ 's neighbors; $\partial i=\{i+1, i-1\}$ in the present case of a circle. A randomly chosen player $i$ chooses the strategy $S_{i}$, conditional on his neighbors' strategies, with the logit probability

$$
\operatorname{Pr}\left(S_{i}\right) \propto \exp \left[\sum_{j \in \partial i} \beta U_{i, j}\right],
$$

where $\beta$ is a non-negative parameter indicating the responsiveness of the utility function to the payoffs. In fact, this parameter reflects the inverse of the variance of the extreme-value of type II shock underlying the logit model. When the variance is very large, $\beta$ is small and the observed utility payoffs are not important for the choice. At the other extreme, when the variance is very small, the shock is relatively less important than the observed payoffs, which in turn dominate the choice. The probability $\operatorname{Pr}\left(S_{i}\right)$ that a player chooses $S_{i}$ in Eq. (4) is recast into the form, after the normalization,

$$
\begin{aligned}
\operatorname{Pr}\left(S_{i}\right)= & \exp \left[\beta \sum_{j \in \partial i}\left(x S_{i} S_{j}+y S_{i}+z S_{j}+w\right)\right] \\
\sum_{S_{i}= \pm 1} \exp \left[\beta \sum_{j \in \partial i}\left(x S_{i} S_{j}+y S_{i}+z S_{j}+w\right)\right] & \frac{\exp \left[\beta \sum_{j \in \partial i}\left(x S_{i} S_{j}+y S_{i}\right)\right]}{\sum_{S_{i}= \pm 1} \exp \left[\beta \sum_{j \in \partial i}\left(x S_{i} S_{j}+y S_{i}\right)\right]}
\end{aligned}
$$




\section{The Exact Solution}

The probability $\operatorname{Pr}\left(S_{i}\right)$ in Eq. (5) that a randomly chosen player $i$ chooses the strategy $S_{i}$ is the same as the probability that a randomly chosen site $i$ chooses $S_{i}= \pm 1$ in the Glauber dynamics (Glauber (1963)). We can use a statistical-mechanical method of analyzing the dynamics.

Let $\{S\}$ denotes the set of a combination of the strategies $\left\{S_{1}, S_{2}, \cdots, S_{N}\right\}$ and let the probability $\operatorname{Pr}(\{S\})$ denote the joint probability that player 1 takes the strategy $S_{1}$, player 2 takes the strategy $S_{2}, \ldots$, and player $N$ takes the strategy $S_{N}$. The cardinality of the set of all possible outcomes for the entire economy, $\{S\}$, is $2^{N}$, because there are $N$ players and two strategies $A$ and $B$. Let us order all possible sets sequentially as

$$
\begin{aligned}
\{S\}_{1} & =\left\{S_{1}=+1, S_{2}=+1, \cdots, S_{N}=+1\right\}, \\
\{S\}_{2} & =\left\{S_{1}=-1, S_{2}=+1, \cdots, S_{N}=+1\right\}, \\
\{S\}_{3} & =\left\{S_{1}=+1, S_{2}=-1, \cdots, S_{N}=+1\right\}, \\
\{S\}_{4} & =\left\{S_{1}=-1, S_{2}=-1, \cdots, S_{N}=+1\right\}, \\
\vdots & \\
\{S\}_{2^{N}} & =\left\{S_{1}=-1, S_{2}=-1, \cdots, S_{N}=-1\right\} .
\end{aligned}
$$

We will occasionally refer to the set of strategies $\{S\}_{\mu}$ as the $\mu$ th configuration and denote it simply as $\mu$, such as in $\operatorname{Pr}(\mu)$. Suppose that configuration $\mu$ is given by $\{S\}_{\mu}=$ $\left\{S_{1}, \cdots, S_{i}, \cdots, S_{N}\right\}$ and configuration $\nu$ is given by $\{S\}_{\nu}=\left\{S_{1}, \cdots, S_{i}^{\prime}, \cdots, S_{N}\right\}$. The difference is the strategy of the player $i$ only, $S_{i}^{\prime} \neq S_{i}$. To transit from configuration $\mu$ to configuration $\nu$, player $i$ is randomly chosen with probability $1 / N$ to update his strategy from $S_{i}$ to $S_{i}^{\prime}$. Let $\mathbf{T}$ denote the transition matrix across the $2^{N}$ configurations. The transition probability $T_{\mu \nu}$ from the configuration $\mu$ to the configuration $\nu$ is, thus, given by

$$
T_{\mu \nu}=\frac{1}{N} \frac{\exp \left[\beta \sum_{j \in \partial i}\left(x S_{i}^{\prime} S_{j}+y S_{i}^{\prime}\right)\right]}{\sum_{S_{i}^{\prime}= \pm 1} \exp \left[\beta \sum_{j \in \partial i}\left(x S_{i}^{\prime} S_{j}+y S_{i}^{\prime}\right)\right]} .
$$

Let $\mathbf{P}$ denote the vector

$$
\mathbf{P}=\left(\operatorname{Pr}(1), \operatorname{Pr}(2), \cdots, \operatorname{Pr}\left(2^{N}\right)\right),
$$

$\mathbf{P}(t)$ denote $\mathbf{P}$ as of time $t$. The vector $\mathbf{P}(t)$ is updated by the transition matrix $\mathbf{T}$ such that $\mathbf{P}(t+1)=\mathbf{P}(t) \mathbf{T}$. We will characterize the probabilities that different strategies prevail at the steady state, which we identify as the equilibrium in the present paper. The steady state $\mathbf{P}_{\mathrm{ss}}$ is the vector to which $\mathbf{P}$ converges when the numbers of updates of strategies tends to infinity. In Appendix A, we will derive $\operatorname{Pr}_{\mathrm{ss}}\left(S_{1}, S_{2}, \cdots, S_{N}\right)$ and show their existence and uniqueness. Our treatment utilizes the fact the dynamics in our model are equivalent to the Glauber dynamics after transformation (2).

The steady state probability of strategies is given by

$$
\operatorname{Pr}_{\mathrm{SS}}\left(S_{1}, \cdots, S_{N}\right)=\frac{\exp \left[\beta H\left(S_{1}, \cdots, S_{N} ; a, b, c, d\right)\right]}{Z(\beta, a, b, c, d)}
$$


where $H$ and $Z$ are defined by

$$
\begin{aligned}
& H\left(S_{1}, \cdots, S_{N} ; a, b, c, d\right) \equiv x \sum_{i=1}^{N} S_{i} S_{i+1}+y \sum_{i=1}^{N} S_{i}, \\
& Z(\beta, a, b, c, d) \equiv \sum_{\{S\}} \exp \left[\beta H\left(S_{1}, \cdots, S_{N} ; a, b, c, d\right)\right]
\end{aligned}
$$

and $\{S\}$ denotes the set of all the possible combinations of the strategies $\left\{S_{1}= \pm 1, S_{2}=\right.$ $\left.\pm 1, \cdots, S_{N}= \pm 1\right\}$.

We will use the transfer-matrix method (see, e.g., Nishimori and Ortiz (2011); Brézin (2010); Baxter (1982)) developed in statistical mechanics in order to obtain the exact strategy probability, the strategy correlation function, and the expected utility. Details are given in Appendix B. We thereby arrive at the following three propositions.

\subsection{Players' Strategy Choice Probabilities}

We have the following proposition concerning the probability that a player chooses a strategy. A derivation is provided in Appendix B.2.

Proposition 1. The probability $p_{A}$ that a player takes the strategy $A$ in the steady state is exactly given by

$$
p_{A}=\frac{\exp \left(\beta \frac{a-b-c+d}{4}\right) \sinh \left(\beta \frac{a+b-c-d}{4}\right)}{2 \sqrt{\exp \left(\beta \frac{a-b-c+d}{2}\right) \cosh ^{2}\left(\beta \frac{a+b-c-d}{4}\right)-2 \sinh \left(\beta \frac{a-b-c+d}{2}\right)}}+\frac{1}{2}
$$

in the limit as $N \rightarrow \infty$.

The sign of $a+b-c-d$ determines risk dominance. Our rationale for considering the limit for $N \rightarrow \infty$ is as follows. We are interested in "emergent" phenomena when many players interact spatially. We underscore that to the best of our knowledge, the exact solution of this kind of model may be obtained in cases of circle and line topologies only.

\subsection{Strategy Correlation Function}

Next we investigate the stochastic interdependence of strategies at different graph-distances from one another. We do so by deriving the strategy correlation function $C(r)$, where $r$ denote the graph-distance between the two players. The strategy correlation function $C(r)$ over distance $r$ is defined by

$$
C(r) \equiv E\left[\left(S_{i}-E\left[S_{i}\right]\right)\left(S_{i+r}-E\left[S_{i+r}\right]\right)\right]=E\left[S_{i} S_{i+r}\right]-E[S]^{2} .
$$

An intuitive interpretation of the strategy correlation function $C(r)$ is as follows. Suppose that shops choose a price between a high and a low price. Provided that a shop chooses the low price, the probability that another shop in the distance $r$ from the first shop may choose the low price is given by the strategy correlation function $C(r)$.

We now have the following proposition, whose derivation is explained in detail in Appendix B.3. 
Proposition 2. The correlation function $C(r)$ of the strategies of players at graph distance $r$ is given by

$$
C(r)=\sin ^{2}(2 \phi)\left(\frac{\lambda_{-}}{\lambda_{+}}\right)^{r}
$$

in the limit $N \rightarrow \infty$, where $\phi$ and $\lambda_{ \pm}$are defined by

$$
\begin{aligned}
\cot (2 \phi) & =\exp \left[\beta \frac{a-b-c+d}{2}\right] \sinh \left(\beta \frac{a+b-c-d}{4}\right) \\
\lambda_{ \pm} & =\exp \left[\beta \frac{a-b-c+d}{4}\right] \cosh \left(\beta \frac{a+b-c-d}{4}\right) \\
& \pm \sqrt{\exp \left[\beta \frac{a-b-c+d}{2}\right] \cosh ^{2}\left(\beta \frac{a+b-c-d}{4}\right)-2 \sinh \left(\beta \frac{a-b-c+d}{2}\right) .}
\end{aligned}
$$

The strategy correlation $C(r)$ of players' strategies at graph distance $r$ decays as $\left(\lambda_{-} / \lambda_{+}\right)^{r}$.

Note that by construction $\left(\frac{\lambda_{-}}{\lambda_{+}}\right)^{r}$ tends to 0 , as $r \rightarrow \infty$. Therefore the correlation $C(r)$ attenuates spatially when the number of agents is large.

\subsection{Expected Utility}

The proposition that follows reports the solution for the expected utility of the game for the typical player. The derivation is given in Appendix B.4.

Proposition 3. The expected utility $E[U]$ is given by

$$
E[U]=\frac{(a-b-c+d)}{2}\left[\cos ^{2}(2 \phi)+\left(\frac{\lambda_{-}}{\lambda_{+}}\right) \sin ^{2}(2 \phi)\right]+(a-d) \cos (2 \phi)+\frac{(a+b+c+d)}{2}
$$

in the limit as $N \rightarrow \infty$.

\subsection{The model on a Line}

So far, we have solved the model with the players located on a circle. The following question naturally arises: what if the spatial structure retains the one-dimension but is construed on a line? The line topology, illustrated in Fig. 2, is conceptually very similar to the circle, with the only difference of the players at either end. We show that the spatial structure of either a circle or a line leads to same results in the limit as $N \rightarrow \infty$. Suppose that there are $N$ players and the player 1 and the player $N$ are on the ends of the line. They interact only with the player 2 and the player $N-1$, respectively. It turns out that with many players, the agents at either end have vanishing influence. Hence, we are concerned with players deep within a line, when $N \rightarrow \infty$.

Regardless of whether the spatial structure is a circle or a line, the probability $p_{A}$ that a player chooses strategy $A$, the strategy correlation function $C(r)$, and the expected utility $E[U]$ are the same in the limit as $N \rightarrow \infty$. The details of the derivations are given in Appendix C. 


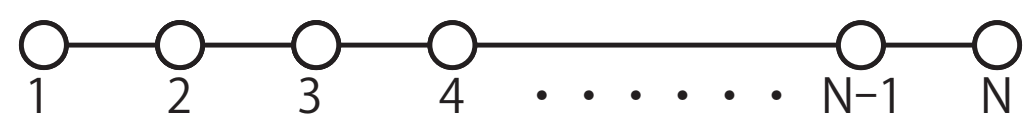

Figure 2: Players are set on a line and play games with adjacent players only.

\subsection{Comparison with the Mean-field Approximation}

Local interaction Logit games have been solved by mean-field approximation in many studies (e.g., those referred in the introduction). We comment here on the relationship between the exact solution and a mean-field approximate solution. A mean-field approximation is the approximation in which the strategies $S$ of adjacent players are replaced by the mean strategy $E[S]$ of all players. In the mean-field approximation, the mean strategy $E[S]$ is given self-consistently by the following relation

$$
E[S]=\tanh \left[\beta\left(\frac{a-b-c+d}{2} E[S]+\frac{a+b-c-d}{4}\right)\right] ;
$$

see, for example, Baxter (1982); Brézin (2010); Nishimori and Ortiz (2011). This is the central equilibrium condition in the interactive discrete choice model [ Brock and Durlauf (2001)]. See also section 5 below.

The mean-field approximation does not take account of details of the underlying spatial structure. This is an instance of a more general property known as universality. See Durlauf (2004). Therefore, the mean-field approximation generally works well in high-dimensional settings, but poorly in low-dimensional ones. In contrast, the exact solution of the game is available only in one dimension as of now. Since in the present study we are interested in the effect of the spatial structure, we chose to work in one dimension and employ the method of the exact solution. The mean-field approximation would produce quite a different result in the present case of one dimension. Indeed, the above condition admits in general three fixed points of the hyperbolic tangent function, which are identified as multiple equilibria; see Brock and Durlauf (2001) and Ioannides (2006).

\subsection{The limits $\beta \rightarrow 0$ and $\beta \rightarrow \infty$}

The parameter $\beta$ indicates the sensitivity of the logit probabilities to the payoff from different strategies. It actually reflects the dispersion of the underlying shocks that give rise to logit probabilities: $\beta \rightarrow 0$ expresses that the variance of the extreme value distributed random shocks that give rise to the logit probabilities tends to infinity. In that case, the probability that a player chooses strategy $A$ is given by $p_{A}=1 / 2$ - the payoffs are irrelevant. The strategy correlation function is given by $C(r)=0$. The corresponding expected utility is given by $E[U]=(a+b+c+d) / 4$.

In the other extreme, $\beta \rightarrow \infty$, the variance of the random shocks is equal to zero, resulting in a deterministic choice: the probability $p_{A}$ of choosing strategy $A$, the strategy correlation function $C(r)$, and the expected utility $E[U]$ all depend on the signs of $x$ and $y$. The players are perfectly rational and choose the best strategy in the limit. 


\section{The Sensitivity of Strategy Probabilities to $\beta$ in Prisoner's Dilemma and Co- operation Games}

\subsection{The Change in the Probability of Cooperation in Response to Changes in $\beta$}

We study next how the probability that a player chooses cooperation changes in response to changes in $\beta$ in a prisoner's dilemma game. The payoff matrix of prisoner's dilemma game under investigation is given by

$$
\begin{array}{cc}
C & D \\
D
\end{array}\left(\begin{array}{cc}
(e-f, e-f) & (-f, e) \\
(e,-f) & (0,0)
\end{array}\right) .
$$

The strategies $\mathrm{C}$ and $\mathrm{D}$ denote cooperation and defection respectively: $e$ denotes the benefit from cooperation and $f$ the cost for cooperation; both of them are assumed to be positive. Let $p_{C}$ denote the probability that a player chooses the strategy $\mathrm{C}$.

We have

$$
\frac{\partial p_{C}}{\partial \beta}=-\frac{1}{4} f \cosh ^{2}\left(\frac{f \beta}{2}\right)<0 .
$$

Proposition 4. The larger $\beta$ is, the lower the probability $p_{C}$ that a player chooses to cooperate in a prisoner's dilemma game is.

corollary 1. As $\beta \rightarrow \infty$, the probability $p_{C}$ that a player chooses cooperative strategy $C$ goes to 0 in the prisoner's dilemma game.

\subsection{The Change in the Probability of Taking Pareto Efficient Strategy in Response to $\beta$}

Next we study how the probability that a player chooses a Pareto efficient strategy changes in response to the increase in $\beta$ in a cooperation game. The payoff matrix prisoner's dilemma game under investigation is given by

$$
\begin{gathered}
P \\
P \\
P\left(\begin{array}{cc}
(a, a) & (0, c) \\
(c, 0) & (d, d)
\end{array}\right),
\end{gathered}
$$

5 where $a>d>c>0$ and $a<c+d$ hold true. The strategy pair $(\mathrm{P}, \mathrm{P})$ is the Pareto efficient one, where strategy $\mathrm{R}$ is risk dominant. The strategies $\mathrm{P}$ and $\mathrm{R}$ denote Pareto efficient and risk dominant strategies, respectively. Let $p_{P}$ denote the probability that a player chooses the Pareto efficient strategy P.

We have

$$
\frac{\partial p_{P}}{\partial \beta}=\frac{e^{-\frac{1}{4} \beta(a-c+d)}\left[2(a-c+d) \sinh \left(\frac{1}{4} \beta(a-c-d)\right)+(a-c-d) \cosh \left(\frac{1}{4} \beta(a-c-d)\right]\right)}{8\left[e^{\frac{1}{2} \beta(a-c+d)} \cosh ^{2}\left(\frac{1}{4} \beta(a-c-d)\right)-2 \sinh \left(\frac{1}{2} \beta(a-c+d)\right)\right]^{3 / 2}}<0,
$$

Proposition 5. The greater $\beta$ is, the lower the probability $p_{P}$ that a player chooses Pareto efficient strategy $P$ in the coordination game is.

corollary 2. As $\beta \rightarrow \infty$, the probability $p_{P}$ that a player chooses Pareto efficient strategy $P$ goes to 0 in the coordination game. 


\section{Relation to social interaction models}

\subsection{Interactions based on Agents' Actual Environments}

Much of the interactive discrete choice literature assumes that agents act by forming expectations over their neighbors' actions. This is the case of Brock and Durlauf $(2000 ; 2001)$ and Ioannides (2006). Those authors employ the self-consistency condition in section 3.5 as an equilibrium condition. With local interactions, however, to be defined shortly, where one knows one's neighbors, it is reasonable to assume that individuals observe their neighbors' actual actions. This is what we take up in this section. By imposing the requirement that agents' optimal decisions be consistent with one another at equilibrium, which constitutes a counterpart of the game-theoretic formulation earlier in the paper, we may, in principle, obtain the probability distribution functions for agents' decisions in terms of fundamentals and of the distributions of the random utility components. This aspect of the model has not been fully explored in the context of the interactive discrete choice literature. ${ }^{4}$

Suppose that individual $i^{\prime}$ s utility function is given by:

$$
U_{i}\left(S_{i} ; \widetilde{S}_{\partial i}\right) \equiv h S_{i}+S_{i} \sum_{j \in \partial i} J_{i j} S_{j}+\epsilon\left(S_{i}\right)
$$

where $\widetilde{S}$ denotes the vector of all agents' decisions, $\left\{S_{1}, \ldots, S_{N}\right\}$, and $\widetilde{S}_{\partial i}$ its subvectors composed of those of $i^{\prime}$ s neighbors, $\partial i \subseteq\{1, \ldots, I\}$, and $J_{i j}$ denote interaction weights. Here, the social component of individual $i$ 's utility is defined as a function of the actual decisions of one's neighbors, $\omega_{i} \sum_{j \in \partial i} J_{i j} \omega_{j}$. Under the assumption that the shocks in (27) are extremevalue distributed, utility maximization by individual $i$, conditional on $\widetilde{S}_{\partial i}$ yields that:

$$
\operatorname{Prob}\left(S_{i}=1 \mid \widetilde{S}_{\partial i}\right)=\frac{\exp \left[\beta\left(2 h+2 \frac{1}{|\nu(i)|} \sum_{j \in \partial i} J_{i j} S_{j}\right)\right]}{1+\exp \left[\beta\left(2 h+2 \frac{1}{|\nu(i)|} \sum_{j \in \partial i} J_{i j} S_{j}\right)\right]}, i=1, \ldots, I
$$

This is a description of each agent's best response conditional on her environment. It coincides with the conditional specification of a Markov random field: the probability distribution of each agent's state depends on those of her neighbors.

A key result from the literature on Markov random fields states that if this is a strictly positive nearest neighbor specification, then there exists a single probability distribution function for the state of the economy $\widetilde{S}$, known as global phase, which is consistent with the local specification [Kindermann and L.Snell (1980)]. The global phase is a Markov random field, and in fact every Markov random field is equivalent to a Gibbs state for some single nearest neighbor potential [ Kindermann and L.Snell (1980) ].

Equilibrium with social interactions when agents know the actual decisions of their neighbors thus have very different structure than those of the Brock-Durlauf model. Being described by means of a probability distribution function that coincides with the global phase of the respective Markov random field, this distribution is characterized by more general dependence than dependence on each agent's neighbors.

\footnotetext{
${ }^{4}$ This is alluded to but examined by Ioannides (2006) only in the dynamic case; see ibid., sections 3.1, 3.1.1 and 3.12. It is presented in Ioannides (2004), sections 3, 3.1.
} 
The two interactions topologies that are particularly interesting to us are interactions over a circle and along a line, as we have discussed earlier in the paper. The former may be referred to as "cyclical interaction as an one-dimensional nearest-neighbor Ising model;" the latter interaction as an "one-dimensional nearest-neighbor Ising model along a line." The solutions corresponding to these specific topologies are exactly analytically equivalent to the solutions obtained earlier in the paper in the asymptotic case for $N \rightarrow \infty$; see Eq. (4). Specifically,

$$
\operatorname{Prob}(\tilde{S}) \sim \exp \left[\beta J \sum_{j=1}^{N} S_{j} S_{j+1}+\beta h \sum_{j=1}^{N} S_{i}\right],
$$

where the normalizing constant is derived in Ioannides (2004), section 3.1 and App. 8.7, and solved for explicitly below in Appendix B. The case of interactions along the line is adapted in the obvious way. As it has already been indicated above, they differ from the Brock-Durlauf setting: the exact solutions for agents' equilibrium behavior that we obtain do not involve fixed points. ${ }^{5}$

\subsection{An Econometric Interpretation}

The model of discrete decisions with social interactions, when individuals' utilities depend on their neighbors' actual decisions, admits an interpretation as an econometric model of simultaneous equations involving discrete decisions. Specifically, if the utility functions of agents $i=1, \ldots, N$, as defined in (27), are interpreted as indicator functions for the discrete choices of the logit models, then the discrete choice probabilities, given by (29), are the corresponding reduced forms for the structural system of simultaneous equations of the logit type (28).

The earlier literature on structural models of discrete choice, such as Schmidt (1981) and others, emphasizes conditions for "internal consistency" or "coherency." Such conditions guarantee that given the values of exogenous variables, observed and unobserved, unique values for the dependent variables are implied and the associated likelihood functions are well defined.

We may think of decisions by an interacting group of $N$ agents with a more general interaction topology. The counterpart of (27), may be written in concise vector form as follows:

$$
\widetilde{S}=\mathbf{1}\left[2 h \mathbf{I}+2 \mathbf{N}^{-1} \boldsymbol{\Gamma} \tilde{S}+\widetilde{\varepsilon}\right],
$$

where $\mathbf{I}$ denote an $N$-vector of 1 's, $\mathbf{N}^{-1}$, the inverse of the matrix of each agent's neighbors, $\boldsymbol{\Gamma}$, the matrix of interaction coefficients which subsumes the adjacency matrix of the interaction topology, an $N \times N$ matrix and the $N$-column vector $\tilde{\varepsilon}$ is defined as the difference of $2 N$ independently and identically type I extreme-value distributed random variables, $\varepsilon_{i}=\epsilon_{i}(1)-$ $\epsilon_{i}(-1)$, written as a column vector, $\tilde{\varepsilon} \equiv \tilde{\epsilon}(1)-\tilde{\epsilon}(-1)$, and $\mathbf{1}[\mathcal{R}]$ is a $N$ - vector indicator function of the $N$-vector $\mathcal{R}$, with its $i$ th element equal to 1 , if the $i$ th element of $\mathcal{R}, \mathcal{R}_{i}>0$, and is equal to -1 , otherwise. Equ. (30) represents a nonlinear spatial autoregressive model for discrete endogenous variables.

The consistency conditions proposed by Schmidt (1981) and applied to (30) reduce to the condition that the model be recursive [ ibid., Condition 12.6, p. 429 ]. It may be shown

\footnotetext{
${ }^{5}$ They do involve an auxiliary variable whose values is defined in terms of the root of Eq. (B.16).
} 
the system of simultaneous discrete choice equations (30) is not recursive for the cases of complete, cyclical, and interaction along the line topologies. The present paper establishes that interactive discrete choice models that are defined in those topologies and with agents' being assumed of acting under full knowledge of their neighbors' decisions are isomorphic to spatial logit response games.

\subsection{Dynamic Analysis of Social Interactions}

We may move to a dynamic analysis with social interactions in nonlinear settings by assuming that agents make decisions with knowledge of the actual state of their neighbors in the preceding period, given an arbitrary interaction topology. That is:

$$
\widetilde{S}_{t}=\mathbf{1}\left[2 h \mathbf{I}+2 \mathbf{N}^{-1} \boldsymbol{\Gamma} \widetilde{S}_{t-1}+\tilde{\varepsilon}\right] .
$$

Ioannides (2006) discusses how the stationary distribution associated with this model may be obtained. Ioannides (2006) also reports an explicit solution for the case of interactions defined on a $d$-dimensional torus, which are translation-invariant and symmetric, a result that rests on Bigelis (1999).

\section{Conclusion}

We have solved in closed form a general two-strategy symmetric-payoff logit-response game with one-dimensional spatial structure, without appealing to a mean-field approximation. Unlike the mean-field approximation of discrete response models, which does not take account of details of the underlying spatial structure, our approach produces an exact solution that does fully depend on network topology. The game we study can analyze several models, which can be interpreted as games with spatial structures, ranging from pure social interactions to retailers competing with their neighbors. A spatial structure is very important in coordination games. The propagation in linguistic innovations and changes in languages and cultures, for example, over the ages in Europe may be explained as spatial coordination games. Changes in languages and cultures diffuse and propagate spatially.

We solve the logit response game with spatial structure by introducing a transformation that maps from the two-strategy spatial logit response games to the Ising-spin interaction model. The transformation makes the dynamics of the game equivalent to the Glauber dynamics of statistical mechanics. We then apply the transfer-matrix method, which is a common tool of statistical mechanics, and obtain the exact solution of the game. We investigate the steady state, which is interpreted as the equilibrium concept in the present paper. We derive the strategy choice probabilities, the strategy correlation function $C(r)$ as a function of the graph-distance $r$ between players, and the expected utility $E[U]$ of the game, an exact measure of players' welfare exactly in closed-form. We study the sensitivity of these endogenous objects to a key parameter of the model. The strategy correlation function $C(r)$ gives the stochastic interdependence across the strategies adopted by other players at distance $r$,

and show that the effects decay geometrically. We show that the greater the sensitivity of the strategy choice probabilities to the payoffs is, that is the parameter $\beta$, the smaller the probability of adopting a cooperative strategy in a prisoner's dilemma game is. We also show that the greater $\beta$ is, the smaller the probability of taking Pareto efficient strategy in the coordination game is, tending to 0 as $\beta \rightarrow \infty$. Unlike mean-field approximations of spatial 
logit response models whose equilibria are characterized by fixed points of the hyperbolic tangent function, our solutions are obtained in closed form. We establish an equivalence between spatial logit response games and social interactions models with agents observing their actual environments, discuss its econometric characterization, and develop its dynamic counterpart.

*Acknowledgement. Konno expresses gratitude to Naomichi Hatano, Shin-ichi Hanada, and Takatoshi Tabuchi for helpful comment and discussions and to Japan Society for the Promotion of Science for financial support.

This research was partly supported by the grants-in-aid from 21st-Century Culture and Science Foundation, Seimeikai, and Japan Society for the Promotion of Science.

\section{Appendix A. The Probability of Strategies in the Steady State}

The present Appendix explains how we may obtain the steady-state probability of adopting different strategies. Our discussion is equivalent to the detailed balance condition of the Metropolis method (Metropolis et al. (1953)), the Gibbs sampler (the heat-bath method), and the Markov Chain Monte Carlo (MCMC). They are basic results for reversible stochastic processes [Kelly (1979); Blume (1993)].

We show the existence and uniqueness of the steady state below. $\operatorname{Pr}_{\mathrm{ss}}(\mu)$ denotes the $\mu$ th component of the steady state $\mathbf{P}_{\mathrm{ss}}$, that is the probability of the set of strategies $\{S\}_{\mu}$ in the steady state, which is also referred to as the steady-state probability of strategies $\{S\}_{\mu}$. Note that in the steady state, the strategy of each player is not stationary but the probability of the strategies is stationary. This steady state is interpreted as the equilibrium in the present paper.

We show that the steady-state probability is given by

$$
\operatorname{Pr}_{\mathrm{ss}}(\mu)=\frac{\exp [\beta H(\mu ; a, b, c, d)]}{Z(\beta, a, b, c, d)},
$$

where $H$ and the $Z$ called partition function are respectively given by

$$
\begin{aligned}
H(\mu ; a, b, c, d) & \equiv H\left(\{S\}_{\mu} ; a, b, c, d\right)=x \sum_{i=1}^{N} S_{i} S_{i+1}+y \sum_{i=1}^{N} S_{i}, \\
Z(\beta, a, b, c, d) & \equiv \sum_{\mu} \exp [\beta H(\mu ; a, b, c, d)],
\end{aligned}
$$

and $\sum_{\mu}$ denotes the summation over all possible $2^{N}$ sets of strategies $\{S\}_{\mu}$. Note that $x$ and $y$ in Eq. (A.2) are functions of $a, b, c$, and $d$ as are defined in Eq. (12). The expectation in the steady state is therefore given by

$$
\begin{aligned}
E[Q] \equiv & =\sum_{\mu} \operatorname{Pr}_{\mathrm{ss}}(\mu) Q(\mu) \\
& =\frac{1}{Z(\beta, a, b, c, d)} \sum_{\mu} Q(\mu) \exp [\beta H(\mu ; a, b, c, d)],
\end{aligned}
$$

for an arbitrary quantity $Q$. We occasionally let $H(\mu)$ denote $H\left(\{S\}_{\mu} ; a, b, c, d\right)$ and $Z$ denote $Z(\beta, a, b, c, d)$. 
After an update of strategies, $\mathbf{P}(t)$ changes to $\mathbf{P}(t+1)$. Let $\mathbf{T}$ denote the transition matrix given by the update rule from the state $\mathbf{P}(t)$ changes to $\mathbf{P}(t+1)$ as defined in Section 3 . The element $T_{\nu \mu}$ of the matrix $\mathbf{T}$ is the transition probability that the system hops from the $\nu$ th configuration (the $\nu$ th set of the strategies $\{S\}_{\nu}$ ) to the $\mu$ th configuration in a update. The update of the strategies is then described by

$$
\mathbf{P}(t+1)=\mathbf{P}(t) \mathbf{T} .
$$

As the update progresses, the probability of strategies converges to the unique steady state $\mathbf{P}_{\text {ss }}$ from any initial state $\mathbf{P}(0)$ as

$$
\lim _{n \rightarrow \infty} \mathbf{P}(0) \mathbf{T}^{n}=\mathbf{P}_{\mathrm{ss}}
$$

The steady state $\mathbf{P}_{\mathrm{ss}}$ exists and is unique thanks to the Perron-Frobenius theorem, because $\left(T^{n}\right)_{\mu \nu}>0, \forall \mu, \nu, \exists n$ for the transition matrix $\mathbf{T}$ under consideration in the present paper.

The steady state $\mathbf{P}_{\mathrm{ss}}$ satisfies

$$
\mathbf{P}_{\mathrm{sS}} \mathbf{T}=\mathbf{P}_{\mathrm{sS}}
$$

or

$$
\sum_{\nu} \mathbf{P}_{\mathrm{ss}}(\nu) T_{\nu \mu}=\mathbf{P}_{\mathrm{ss}}(\mu), \forall \mu
$$

which is followed by

$$
\sum_{\nu(\neq \mu)} \mathbf{P}_{\mathrm{SS}}(\nu) T_{\nu \mu}+\mathbf{P}_{\mathrm{SS}}(\mu)\left(1-\sum_{\nu(\neq \mu)} T_{\mu \nu}\right)=\mathbf{P}_{\mathrm{SS}}(\mu),
$$

because the transition matrix must satisfy

$$
\sum_{\nu=1}^{2^{N}} T_{\mu \nu}=1
$$

Hence, we obtain

$$
\sum_{\nu(\neq \mu)} \mathbf{P}_{\mathrm{Ss}}(\nu) T_{\nu \mu}=\sum_{\nu(\neq \mu)} \mathbf{P}_{\mathrm{ss}}(\mu) T_{\mu \nu} .
$$

It is generally not easy to obtain the steady-state solution of Eq. (A.11) for a given transition matrix T. However, it is much easier to solve a sufficient condition of Eq. (A.11),

$$
\mathbf{P}_{\mathrm{ss}}(\nu) T_{\nu \mu}=\mathbf{P}_{\mathrm{ss}}(\mu) T_{\mu \nu}, \forall \mu, \nu,
$$

which is called the detailed balance condition in the field of statistical physics. Equation (A.12) means that the probability flow from any configuration into any other configuration is equal to the probability flow in the opposite direction. Since the steady state is known to be unique, a solution that satisfies the detailed balance condition (A.12) must be 
the unique steady-state solution of Eq. (A.11). Noting that the transition matrix given in Eq. (11) satisfies

$$
\frac{T_{\mu \nu}}{T_{\nu \mu}}=\frac{\exp [\beta H(\nu)]}{\exp [\beta H(\mu)]}
$$

we find that any components of the steady state $\mathbf{P}_{\mathrm{ss}}$ should satisfy

$$
\frac{\mathrm{P}_{\mathrm{sS}}(\nu)}{\mathrm{P}_{\mathrm{ss}}(\mu)}=\frac{\exp [\beta H(\nu)]}{\exp [\beta H(\mu)]}
$$

In view of the normalization condition

$$
\sum_{\mu=1}^{2^{N}} \mathrm{P}_{\mathrm{ss}}(\mu)=1,
$$

we arrive at the solution (A.1).

\section{Appendix B. Transfer-Matrix Calculation of the Exact Solution}

In the present Appendix, we show a method of calculating the summation over $\mu$ in the partition function $Z$ in Eq. (A.3). We then obtain for the steady state, closed-form expressions of (i) the probability that a player choose a strategy, (ii) the strategy correlation function, and (iii) the expected utility. The method that we use here is the transfer-matrix method developed for statistical-mechanical models in one spatial dimension (see, e.g., Baxter (1982); Brézin (2010); Nishimori and Ortiz (2011)).

Appendix B.1. Partition function $Z$

Let us define the matrix $\mathbf{V}$ such that the matrix elements $V\left(S, S^{\prime}\right)$ are given by

$$
V\left(S, S^{\prime}\right) \equiv \exp \left[\beta x S S^{\prime}+\frac{1}{2} \beta y\left(S+S^{\prime}\right)\right] .
$$

In other words, we have

$$
\begin{aligned}
& V(+1,+1)=\exp (\beta x+\beta y), \\
& V(+1,-1)=\exp (-\beta x), \\
& V(-1,+1)=\exp (-\beta x), \\
& V(-1,-1)=\exp (\beta x-\beta y),
\end{aligned}
$$

where $S=+1$ indicates the first row and column of the matrix, while $S=-1$ indicates its second row and column. The matrix representation is therefore given by

$$
\mathbf{V}=\left(\begin{array}{cc}
e^{\beta x+\beta y} & e^{-\beta x} \\
e^{-\beta x} & e^{\beta x-\beta y}
\end{array}\right)
$$

We begin with noting the identity

$$
\exp \left[\beta H\left(\{S\}_{\mu}\right)\right]=V\left(S_{1}, S_{2}\right) V\left(S_{2}, S_{3}\right) \cdots V\left(S_{N-1}, S_{N}\right) V\left(S_{N}, S_{1}\right) .
$$


Therefore, the partition function (A.3) is given by

$$
Z=\sum_{\mu} V\left(S_{1}, S_{2}\right) V\left(S_{2}, S_{3}\right) \cdots V\left(S_{N-1}, S_{N}\right) V\left(S_{N}, S_{1}\right)
$$

The summation $\sum_{\mu}$ here is decomposed to

$$
\sum_{\mu}=\sum_{S_{1}= \pm 1} \sum_{S_{2}= \pm 1} \cdots \sum_{S_{N}= \pm 1}
$$

Therefore, Eq. (B.8) indeed takes the form of matrix multiplication:

$$
Z=\sum_{\mu_{1}=1,2} \sum_{\mu_{2}=1,2} \cdots \sum_{\mu_{N}=1,2} V_{\mu_{1} \mu_{2}} V_{\mu_{2} \mu_{3}} \cdots V_{\mu_{N-1} \mu_{N}} V_{\mu_{N} \mu_{1}}
$$

which is reduced to

$$
Z=\operatorname{Tr} \mathbf{V}^{N}
$$

We can calculate the expression (B.11) easily by diagonalizing the matrix V. Since the matrix $\mathbf{V}$ is symmetric, we can diagonalize it by means of an orthogonal matrix $\mathbf{O}$ in the form

$$
\mathbf{O}^{-1} \mathbf{V O}=\mathbf{D} \equiv\left(\begin{array}{cc}
\lambda_{+} & 0 \\
0 & \lambda_{-}
\end{array}\right)
$$

where $\left|\lambda_{+}\right|>\left|\lambda_{-}\right|$and $\lambda_{+}>0$ We can exclude the case of degenerate eigenvalues thanks to the Perron-Frobenius theorem. We then have the partition function in the form

$$
Z=\operatorname{Tr} \mathbf{V}^{N}=\operatorname{Tr} \mathbf{D}^{N}=\lambda_{+}{ }^{N}+\lambda_{-}{ }^{N} .
$$

The explicit expressions of $U$ and $\lambda_{ \pm}$are given by

$$
\begin{aligned}
U & =\left(\begin{array}{cc}
\cos \phi & -\sin \phi \\
\sin \phi & \cos \phi
\end{array}\right), \\
\lambda_{ \pm} & =e^{\beta x} \cosh (\beta y) \pm \sqrt{e^{2 \beta x} \cosh ^{2}(\beta y)-2 \sinh (2 \beta x)},
\end{aligned}
$$

where $\phi$ is defined by the following equation.

$$
\cot (2 \phi)=e^{2 \beta x} \sinh (\beta y)
$$

In the limit $N \rightarrow \infty$, the expression (B.13) is dominated by the first term because $\left|\lambda_{+}\right|>\left|\lambda_{-}\right|$ and $\lambda_{+}>0$. We thereby have

$$
\lim _{N \rightarrow \infty} Z \sim \lambda_{+}{ }^{N}
$$




\section{Appendix B.2. Probability of a strategy}

We now calculate the probability $p_{A}$ that a player chooses the strategy $A$. Since $S_{i}= \pm 1$ corresponds to the strategies $A$ and $B$ of the player $i$, respectively, we have

$$
p_{A}=\frac{1}{2}(E[S]+1)
$$

where

$$
S \equiv \frac{1}{N} \sum_{i=1}^{N} S_{i}
$$

and $E[\cdots]$ is the expectation in the steady state, as was defined in Eq. (A.4).

We can also calculate $E[S]$ by the transfer-matrix method. Its definition in conjunction with the identity (B.7) is followed by

$$
\begin{aligned}
E\left[S_{i}\right]=\frac{1}{Z} \sum_{\{S\}_{\mu}} & V\left(S_{1}, S_{2}\right) V\left(S_{2}, S_{3}\right) \cdots \\
& \cdots V\left(S_{i-1} S_{i}\right) S_{i} V\left(S_{i}, S_{i+1}\right) \cdots \\
& \cdots V\left(S_{N-1}, S_{N}\right) V\left(S_{N}, S_{1}\right) .
\end{aligned}
$$

Let us then define the matrix $\mathbf{M}$ such that the matrix elements are given by

$$
M\left(S, S^{\prime}\right)=S \delta_{S, S^{\prime}}
$$

Its matrix representation is given by

$$
\mathbf{M}=\left(\begin{array}{cc}
1 & 0 \\
0 & -1
\end{array}\right)
$$

Then we can replace $S_{i}$ in Eq. (B.20) with $M\left(S_{i}, S_{i}^{\prime}\right)$. After the same reasoning as in Eqs. (B.7)-(B.11), we obtain

$$
\begin{gathered}
E\left[S_{i}\right]=\frac{1}{Z} \sum_{\{S\}_{\mu}, S_{i}^{\prime}} V\left(S_{1}, S_{2}\right) V\left(S_{2}, S_{3}\right) \cdots \\
\cdots V\left(S_{i-1} S_{i}\right) M\left(S_{i}, S_{i}^{\prime}\right) V\left(S_{i}^{\prime}, S_{i+1}\right) \cdots \\
\cdots V\left(S_{N-1}, S_{N}\right) V\left(S_{N}, S_{1}\right) \\
=\frac{1}{Z} \operatorname{Tr}\left(V^{i-1} M V^{N-i+1}\right)=\frac{1}{Z} \operatorname{Tr}\left(M V^{N}\right) .
\end{gathered}
$$

This indeed proves the uniformity of the space

$$
E[S]=E\left[S_{i}\right], \forall i .
$$

By diagonalizing the matrix $\mathbf{V}$ by using matrix $\mathbf{O}$ again, we have

$$
E[S]=\frac{1}{Z} \operatorname{Tr}\left(\mathbf{O}^{-1} \mathbf{M O D}^{N}\right) .
$$


Using the identity

$$
\mathbf{O}^{-1} \mathbf{M O}=\left(\begin{array}{cc}
\cos (2 \phi) & -\sin (2 \phi) \\
-\sin (2 \phi) & -\cos (2 \phi)
\end{array}\right)
$$

we arrive at

$$
E[S]=\frac{1}{\lambda_{+}{ }^{N}+\lambda_{-}{ }^{N}}\left(\lambda_{+}{ }^{N}-\lambda_{-}{ }^{N}\right) \cos (2 \phi)
$$

In the limit $N \rightarrow \infty$, we find

$$
\lim _{N \rightarrow \infty} E[S]=\cos (2 \phi) .
$$

The explicit expression of $E[S]$ in the limit $N \rightarrow \infty$ is given by

$$
\lim _{N \rightarrow \infty} E[S]=\frac{e^{\beta x} \sinh (\beta y)}{\sqrt{e^{2 \beta x} \cosh ^{2}(\beta y)-2 \sinh (2 \beta x)}} .
$$

Remembering that $x$ and $y$ are functions of the game parameters $a, b, c$, and $d$, we arrive at

$$
\lim _{N \rightarrow \infty} p_{A}=\frac{\exp [\beta(a-b-c+d) / 4] \sinh [\beta(a+b-c-d) / 4]}{2 \sqrt{\exp [\beta(a-b-c+d) / 2] \cosh ^{2}[\beta(a+b-c-d) / 4]-2 \sinh [\beta(a-b-c+d) / 4]}}+\frac{1}{2} .
$$

The sign of $y=a+b-c-d$ determines the risk dominance.

\section{Appendix B.3. Strategy correlation function}

We now calculate the strategy correlation function $C(r)$ over the distance $r$, which is defined in Eq. (7), or

$$
\begin{aligned}
C(r) & \equiv E\left[\left(S_{i}-E\left[S_{i}\right]\right)\left(S_{i+r}-E\left[S_{i+r}\right]\right)\right] \\
& =E\left[S_{i} S_{i+r}\right]-E[S]^{2},
\end{aligned}
$$

where we used the uniformity $E\left[S_{i}\right]=E[S]$. The expectation $E\left[S_{i} S_{i+r}\right]$ can be calculated by the transfer-matrix method again. The same transformation as in Eqs. (B.20)-(B.28) leads to

$$
\begin{aligned}
E\left[S_{i} S_{i+r}\right] & =\frac{1}{Z} \operatorname{Tr}\left(M V^{r} M V^{N-r}\right) \\
& \stackrel{N \rightarrow \infty}{\longrightarrow} \cos ^{2}(2 \phi)+\left(\frac{\lambda_{-}}{\lambda_{+}}\right)^{r} \sin ^{2}(2 \phi) .
\end{aligned}
$$

Therefore, the strategy correlation function $C(r)$ over the distance $r$ is given by

$$
C(r)=\left(\frac{\lambda_{-}}{\lambda_{+}}\right)^{r} \sin ^{2}(2 \phi)
$$


where $\phi$ and $\lambda_{ \pm}$are written in terms of the original parameters as

$$
\begin{aligned}
\cot (2 \phi) & =\exp \left(\beta \frac{a-b-c+d}{2}\right) \sinh \left(\beta \frac{a+b-c-d}{4}\right), \\
\lambda_{ \pm} & =\exp \left(\beta \frac{a-b-c+d}{4}\right) \cosh \left(\beta \frac{a+b-c-d}{4}\right) \\
& \pm \sqrt{\exp \left(\beta \frac{a-b-c+d}{2}\right) \cosh ^{2}\left(\beta \frac{a+b-c-d}{4}\right)-2 \sinh \left(\beta \frac{a-b-c+d}{2}\right) .}
\end{aligned}
$$

\section{Appendix B.4. The expected utility}

The expected utility is given by

$$
\begin{aligned}
E[U] & =E\left[\sum_{j \in \partial i}\left(x S_{i} S_{j}+y S_{i}+z S_{j}+w\right)\right] \\
& =x E\left[S_{i-1} S_{i}\right]+x E\left[S_{i} S_{i+1}\right]+k y E\left[S_{i}\right]+z E\left[S_{i-1}\right]+z E\left[S_{i+1}\right]+k w \\
& =k x E\left[S_{i} S_{i+1}\right]+k(y+z) E[S]+k w,
\end{aligned}
$$

where $k=2$ is the coordination number (the number of the adjacent players). We therefore arrive at

$$
\begin{aligned}
E[U]= & \frac{k(a-b-c+d)}{4}\left(\cos ^{2}(2 \phi)+\frac{\lambda_{-}}{\lambda_{+}} \sin ^{2}(2 \phi)\right) \\
& +\frac{k(a-d)}{2} \cos (2 \phi)+\frac{k(a+b+c+d)}{4} .
\end{aligned}
$$

\section{Appendix C. The Model on a Line}

In the present Appendix, we will solve the model on a line (Fig. 2) by the transfer-matrix method, in order to show that the result does not depend on whether the underlying structure is a circle or a line in the limit $N \rightarrow \infty$. Let us first calculate the partition function $Z$ on a line. The equation is modified from Eq. (B.10) to

$$
Z^{\text {line }}=\sum_{\mu_{1}=1,2} \sum_{\mu_{2}=1,2} \cdots \sum_{\mu_{N}=1,2} \mathbf{V}_{\mu_{1} \mu_{2}} \mathbf{V}_{\mu_{2} \mu_{3}} \cdots \mathbf{V}_{\mu_{N-1} \mu_{N}}
$$

Note that the final matrix element in Eq. (B.10) is missing in Eq. (C.1). We therefore have

$$
Z=\sum_{\mu=1,2} \sum_{\nu=1,2}\left(\mathbf{V}^{N-1}\right)_{\mu \nu}=\left(\begin{array}{ll}
1 & 1
\end{array}\right) \mathbf{V}^{N-1}\left(\begin{array}{l}
1 \\
1
\end{array}\right)
$$

instead of Eq. (B.11). The same diagonalization as in Eq. (B.12) gives

$$
\begin{aligned}
Z^{\text {line }} & =\left(\begin{array}{ll}
1 & 1
\end{array}\right)\left(\begin{array}{cc}
\cos \phi & -\sin \phi \\
\sin \phi & \cos \phi
\end{array}\right)\left(\begin{array}{cc}
\lambda_{+}{ }^{N-1} & 0 \\
0 & \lambda_{-}{ }^{N-1}
\end{array}\right)\left(\begin{array}{cc}
\cos \phi & \sin \phi \\
-\sin \phi & \cos \phi
\end{array}\right)\left(\begin{array}{l}
1 \\
1
\end{array}\right) \\
& =\lambda_{+}{ }^{N-1}\left(1+\sin ^{2} \phi\right)+\lambda_{-}{ }^{N-1}\left(1-\sin ^{2} \phi\right) .
\end{aligned}
$$


In the limit $N \rightarrow \infty$, we thereby have

$$
\lim _{N \rightarrow \infty} Z \sim \lambda_{+}{ }^{N-1}\left(1+\sin ^{2} \phi\right) .
$$

The expectation $E\left[S_{i}\right]$ is similarly given by

$$
E\left[S_{i}\right]=\frac{1}{Z^{\text {line }}}\left(\begin{array}{ll}
1 & 1
\end{array}\right) \mathbf{V}^{i-1} \mathbf{M V}^{N-i}\left(\begin{array}{l}
1 \\
1
\end{array}\right)
$$

instead of Eq. (B.23). The same procedure as Eqs. (B.25)-(B.28) gives

$$
E\left[S_{i}\right]=\left(\begin{array}{ll}
1 & 1
\end{array}\right) \mathbf{O D}^{i-1}\left(\mathbf{O}^{-1} \mathbf{M O}\right) \mathbf{D}^{N-i} \mathbf{O}^{-1}\left(\begin{array}{l}
1 \\
1
\end{array}\right) .
$$

In the limit $i, N \rightarrow \infty$ with $1 \ll i \ll N$, we have

$$
\lim _{\substack{N \rightarrow \infty \\ 1 \ll i \ll N}} \lim _{\substack{i \rightarrow \infty \\ 1 \ll i}} E\left[S_{i}\right]=\cos (2 \phi),
$$

which is identical with Eq. (B.28).

The expectation $E\left[S_{i} S_{i+r}\right]$, which is given on a line by

$$
E\left[S_{i} S_{i+r}\right]=\frac{1}{Z^{\text {line }}}\left(\begin{array}{ll}
1 & 1
\end{array}\right) \mathbf{V}^{i-1} \mathbf{M} \mathbf{V}^{r} \mathbf{M} \mathbf{V}^{N-r-i}\left(\begin{array}{l}
1 \\
1
\end{array}\right)
$$

converges to Eq. (B.32) in the limit $1 \ll i<i+r \ll N$. Therefore, the strategy correlation function $C(r)$ as well as the expected utility $E[U]$ on a line are identical to Eqs. (B.33) and (B.37), respectively.

\section{References}

Anderson, S., De Palma, A., Thisse, J., 1992. Discrete choice theory of product differentiation. MIT Press.

Baxter, R., 1982. Exactly solvable models in statistical physics. Academic Press, London.

Bayer, P., Timmins, C., 2005. On the equilibrium properties of locational sorting models. Journal of Urban Economics 57 (3), $462-477$.

Bigelis, S., E. C. J. L. E. S., 1999. Critical droplets in metastable states of probabilistic cellular automata. Physical Review Letters E 59 (4), 3935-3941.

Blume, L. E., 1993. The statistical mechanics of strategic interaction. Games and Economic Behavior 5 (3), 387-424.

Brézin, E., 2010. Introduction to statistical field theory. Cambridge University Press.

Brock, W., Durlauf, S., 2001. Discrete choice with social interactions. Review Of Economic Studies 68 (2), 235-260.

Chang, S.-K., 2007. A simple asset pricing model with social interactions and heterogeneous beliefs. Journal of Economic Dynamics and Control 31 (4), 1300 - 1325. 
Föllmer, H., 1974. Random economies with many interacting agents. Journal of Mathematical Economics 1 (1), 51-62.

Glauber, R., 1963. Time-dependent statistics of the ising model. Journal of mathematical physics 4, 294.

Hautsch, N., Klotz, S., 2003. Estimating the neighborhood influence on decision makers: theory and an application on the analysis of innovation decisions. Journal of Economic Behavior \& Organization 52 (1), 97 - 113.

Ioannides, Y., 2004. Topologies of social interactions: Conference version. North American Meetings of the Econometric Society, 2004.

Ioannides, Y., 2006. Topologies of social interactions. Economic Theory 28, 559-584.

Ising, E., 1925. Beitrag zur theorie des ferromagnetismus. Zeitschrift fur Physik A Hadrons and Nuclei 31, 253-258.

Kelly, F., 1979. Reversibility and stochastic networks.

Kindermann, R., L.Snell, J., 1980. Markov random fields and their applications. American Mathematical Society, Providence, Rhode Island.

Levy, M., 2008. Stock market crashes as social phase transitions. Journal of Economic Dynamics and Control 32 (1), 137-155.

Luce, R., 1959. Individual choice behavior. Wiley New York.

Lux, T., 1995. Herd behaviour, bubbles and crashes. The Economic Journal 105 (431), 881896.

Lux, T., 1997. Time variation of second moments from a noise trader/infection model. Journal of Economic Dynamics and Control 22 (1), 1-38.

Nishimori, H., Ortiz, G., 2011. Elements of Phase Transitions and Critical Phenomena. Oxford Univ Pr.

Schmidt, P., 1981. Constraints on the parameters in simultaneous tobit and probit models., In Charles F. Manski and Daniel McFadden, eds., Structural Analysis of Discrete Data with Econometric Applications. 422-434.

Sznajd-Weron, K., Sznajd, J., 2000. Opinion Evolution in Closed Community. International Journal of Modern Physics C 11 (6), 1157.

Train, K., 2003. Discrete choice methods with simulation. Cambridge Univ Pr.

Weidlich, W., 1972. The use of statistical models in sociology. Collective Phenomena 1 (1), $51-59$.

Weidlich, W., 1991. Physics and social science-The approach of synergetics. Physics Reports 204 (1), 1-163.

Zanella, G., 2007. Discrete Choice with Social Interactions and Endogenous Memberships. Journal of the European Economic Association 5 (1), 122-153. 